Nephrologe 2010 · 5:91-93

DOI 10.1007/s11560-010-0417-2

๑) Springer-Verlag 2010

\author{
U. Kunzendorf ${ }^{1} \cdot$ U. Heemann ${ }^{2}$ \\ ${ }^{1}$ Klinik für Innere Medizin IV, Nieren- und Hochdruckkrankheiten, \\ Universitätsklinikum Schleswig-Holstein, Campus Kiel \\ ${ }^{2}$ Interdisziplinäres Zentrum für Gefäßerkrankungen, Abteilung für Nephrologie \\ der II. Medizinischen Klinik und Poliklinik, Klinikum rechts der Isar, München
}

\title{
Transplantation und Immunsuppression
}

Die Nierentransplantation bietet Patienten mit einer terminalen Niereninsuffizienz im Vergleich mit den verschiedenen Dialyseverfahren eine längere Lebenserwartung, eine geringere Morbidität und einen erheblich besseren Lebenskomfort. Gerade Kinder profitieren in ihrer körperlichen und geistigen Entwicklung von einer frühzeitigen Transplantation; Erwachsenen erlaubt sie häufig ein nahezu normales Leben - selbst sportliche Spitzenleistungen, wie die des Fußballprofis Ivan Klasnic, sind nach einer erfolgreichen Transplantation wieder möglich. Während das operative Vorgehen in den vergangenen vierzig Jahren nahezu beibehalten wurde, hat sich die Art der Immunsuppression wesentlich geändert. Sicher trägt die Verbesserung der Immunsuppression entscheidend zur Verbesserung der Ergebnisse auf dem Gebiet der Nierentransplantation bei. Aber auch die verbesserte Therapie der kardiovaskulären Komplikationen und der malignen Erkrankungen der Patienten, die aufgrund der Transplantation auf beiden Gebieten ein deutlich höheres Risiko als die Normalbevölkerung aufweisen, sind wesentliche Faktoren des Erfolges.

Bei aller Freude über den Erfolg begründen eine Reihe ungelöster Probleme die weitere intensive Forschung nach neuen immunsuppressiven Medikamenten. (1) So verschlechtert sich die Transplantatfunktion über die Jahre kontinuierlich, sodass Patienten mehrfach transplantiert werden müssen und fast ein Fünftel der Patienten auf der Warteliste bereits einmal transplantiert worden ist. (2) Unverändert bleiben kardiovaskuläre Erkrankungen Haupttodesursache der Transplantierten und ein Grund für die im Vergleich mit der Normalbevölkerung geringere Lebenserwartung. (3) Schließlich entscheiden die Art und das Ausmaß der Immunsuppression, welches Risiko für Infektionen und maligne Erkrankungen der Patient zu tragen hat.

\section{( Eine risikofreie Immunsuppression ist nur durch die Induktion immunologischer Toleranz zu realisieren}

Das Ziel einer risikofreien Immunsuppression ist nur durch die Induktion immunologischer Toleranz zu verwirklichen. Vielleicht gelingt dies, vorerst jedoch muss die Immunsuppression mit den vorhandenen Medikamenten optimiert und das immunsuppressive Repertoire durch neue Medikamente ergänzt werden. Die Autoren dieser Ausgabe nehmen Stellung zu wichtigen Fragen der Immunsuppression. Dabei fließen eigene Erfahrungen und die persönliche Gewichtung der Studienlage mit ein. Besonders bei den Calcineurininhibitoren, Ciclosporin und Tacrolimus, sowie bei den Steroiden - Medikamenten, die seit Jahrzehnten feste Bestandteile der Immunsuppression sind - stellt sich die Frage, wann und wie lange sie eingesetzt werden sollten. Gute Langzeitstudien, die diese Frage eindeutig beantworten, sind rar oder fehlen. Die Immunsuppression nach Transplantation nichtrenaler solider Organe lässt Effekte der immunsuppressiven Medikamente auf die Nieren erkennen, die nach Nierentransplantation immer durch die Immunantwort gegen das Transplantat modifiziert werden. Darü- 
ber hinaus werden neue Medikamente, die sich in der "pipeline" befinden, vorgestellt und auf ihre zukünftige Potenz hin diskutiert. Schließlich gibt die Übersetzung der aktuellen KDIGO-Leitlinien für die Behandlung von Patienten nach Nierentransplantation (Am J Transplant 2009; 9, suppl 3: 1-152) wichtige Informationen zur immunsuppressiven Therapie an die Hand und nennt den Grad der Evidenz.

Die vorliegende Ausgabe von „Der Nephrologe“ zur Immunsuppression nach Nierentransplantation soll dem Leser einen Einblick in den gegenwärtigen Stand der Diskussion zu diesem Thema vermitteln und ermöglichen, anhand der Literaturverzeichnisse Fragen intensiv und gezielt zu bearbeiten.

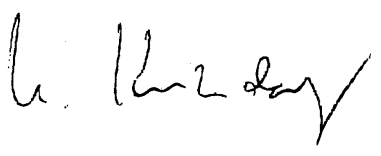

U. Kunzendorf

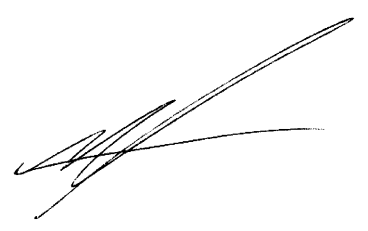

U. Heemann

\section{Korrespondenzadressen \\ Prof. Dr. U. Kunzendorf}

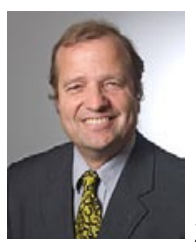

Klinik für Innere Medizin IV, Nieren- und Hochdruckkrankheiten, Universitätsklinikum SchleswigHolstein, Campus Kiel Schittenhelmstraße12, 24105 Kiel kunzendorf@nephro. uni-kiel.de

\section{Prof. Dr. Dr. h.c. U. Heemann}

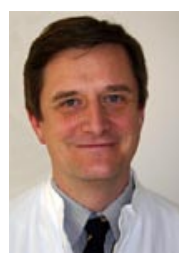

Interdisziplinäres Zentrum für Gefäßerkrankungen, Abteilung für Nephrologie der II. Medizinischen Klinik und Poliklinik, Klinikum rechts der Isar, München Ismaninger Str. 22, 81675 München uwe.heemann@|rz.tum.de
W. Hepp, Michael Koch, K. Konner (Hrsg.) Dialyseshunts

Grundlagen, Chirurgie,

Komplikationen

Steinkopff Verlag 2009, 2. Auflage, 441 S., (ISBN 978-3-7985-1571-0), 99.00 EUR

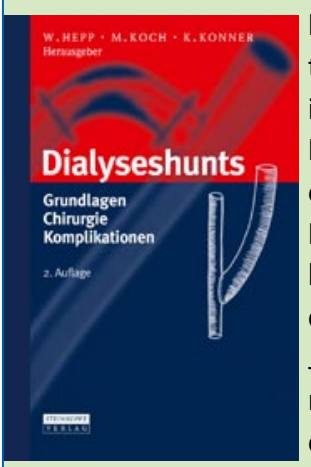

Die Anzahl terminal niereninsuffizienter Patienten, die einen dauerhaften Dialysezugang benötigen, hat in den vergangenen Jahres stetig zugenommen. Ursache der steigenden Zahl neu hin-

zugekommender Dialysepatienten ist eine verbesserte Lebenserwartung der Bevölkerung insgesamt, aber auch die deutlich verbesserte Lebenserwartung der Patienten, die bereits dialysepflichtig sind. Allein diese Tatsache verleiht dem Thema Access Surgery einen wichtigen Stellenwert. Somit ist nach 11 Jahren eine Neuauflage mit inhaltlicher Neuausrichtung des vorliegenden Buches erforderlich geworden. Das Buch greift einen für die Planung eines dauerhaften Dialysezuganges essenziellen Problempunkt auf: die interdisiziplinäre Indikationsstellung, Pflege, Komplikationsmanagement und Diskussion des optimalen Zugangsweges. Die Interdisziplinarität ist somit zu einem wichtigen Eckpfeiler in der Behandlung terminal niereninsuffizienter Patienten geworden, ein Punkt, der zu der Bildung erster interdisziplinärer Shuntzentren geführt hat. Wer das vorliegende Buch liest, erkennt zufort, dass die Behandlung dieser Patienten eine Spezialisierung erfordert, dazu eine langjährige Erfahrung in dem jeweiligen Fachgebiet. Es muss endgültig Vergangenheit sein, dass der jüngste Gefäßchirurg seine technische Expertise bei der Anlage von Shuntzugängen übt! Der Dialysezugang ist die Lebensader des terminal Niereninsuffizienten - von seiner Qualität hängt nicht nur sein Überleben, sondern auch seine Lebensqualität entscheidend ab. Neben der Interdisziplinarität suggerieren die Herausgeber somit ebenfalls eine Korrelation von Behandlungsqualität und Behandlungsquantität. Auch wenn die wissenschaftliche Rechtfertigung für eine Mindestmengendiskussion (die zwischen den Fachgesellschaften aktuell geführt wird) für die Shuntchirurgie bislang nicht schlüssig belegt ist, liegt es nahe, dass eine Konzentration auf Zentren für den Behandlungsablauf und die Ergebnisqualität vorteilhaft ist.

Das vorliegende Buch ist von einer Vielzahl renommierter Autoren unterschiedlicher fachlicher Ausrichtung geschrieben und konzipiert worden. Trotz dieser großen Heterogenität ist es Autoren und Herausgebern gelungen, ein homogenes, ausgewogenes und didaktisch gut strukturiertes Standardwerk zu verfassen, das einen wichtigen Meilenstein für die Shuntchirurgie darstellt. Die Fachliteratur, die sich ausschließlich mit dieser wichtigen Thematik beschäftigt, ist - noch - spärlich. Das vorliegende Werk sollte mentaler Bestandteil jedes Mediziners sein, der sich intensiv mit dieser Thematik beschäftigt.

Sebastian Debus (Hamburg) 
Hier steht eine Anzeige.

算 Springer 